\section{Numerical prediction of temperature and density distributions in selective laser sintering processes}

\author{
Gabriel Bugeda Miguel Cervera \\ and Guillermo Lombera
}

\section{The authors}

Gabriel Bugeda and M iguel Cervera are both with the Civil Engineering Department at the Technical University of Catalonia (UPC), Barcelona, Spain. Materials Technology, National University of Mar del Plata, Mar del Plata, Argentina.

\section{Keywords}

Finite elements, Numerical analysis, Numerical simulation, Rapid prototyping, Selective laser sintering

\section{Abstract}

A finite element model has been developed for the 3D simulation of the sintering of a single track during a selective laser sintering process (SLS). The model takes into account both the thermal and the sintering phenomena involved in the process. Owing to the continuous movement of the laser beam the model takes also into account the transient nature of the problem. This is transformed into a pseudo-static one through a transformation of the coordinates system of the equations. Nevertheless, this transformation introduces a convective term into the heat equations that produces instabilities in the solution. These instabilities have been solved by using a stream upwind Petrov Galerkin (SUPG) strategy together with a shock capturing scheme. Fnally, a fixed point strategy is used for the solution of the analysis. The model has been tested through the solution of some examples.
Guillermo Lombera is at the National Institute of

\section{Introduction}

Selective laser sintering (SLS) is a manufacturing technique which uses a laser beam to sinter material powder in a selective way to produce three dimensional parts. This technique was initially developed at the University of Texas at Austin, and by the companies DTM Corporation, Austin, Texas and BFGoodrich, Brecksville, Ohio. This process consists of three stages (Scherer, 1977a; $1977 b ; 1977 \mathrm{c} ; 1986)$ as it is schematized in Figure 1:

(1) A layer of powder is deposited on the elevator and pressed; each layer of powder is pre-heated before the scanning to minimize the need of induced laser heat.

(2) Laser radiation sinters the powder to form the profile of the section.

(3) The elevator drops through a distance equivalent to the thickness of the section, and the process is repeated until the prototype is completed.

The materials employed are nylon, polycarbonate, ABS, and metal powders. The physical processes associated to this technology include heat transfer and sintering of powder (Ming-Shen et al., 1991 a; 1991b; Weissman and $\mathrm{Hsu}, 1991)$. The sintering models used in this work are based on the analysis of Scherer (1977a; 1997b; 1977c; 1986) and Mackenzie and Shuttleworth (1949). In this analysis, the temperature at each point of the powder bed provides the sintering rate, and the density change is obtained by integration of the sintering rate. Also, the thermal conductivity at each point of the particle bed is a function of the density.

In addition to the thermal and sintering models used in this work there are other possibilities like the models presented by Berzins et al. (1996) and Williams and Deckard (1998). These alternative models could also be integrated in the coupled solution strategy presented at the end of this work.

This research work has been supported by the BRITE/EURAM Proyect "Development of rapid prototyping technologies based on laser sintering" Contract Nr. BRE2-CT 92-0228. The third author would also like to thank CONICET for the grant received.

Special acknowledgment is also due to Dr R.

Rapid Prototyping Journal

Volume 5 - Number 1 - 1999 · pp. 21-26

c ( MCB University Press · ISSN 1355-2546
Codina for his help and suggestions on the solution of the thermal equations. 
Figure 1 Selective laser sintering system Basic Components

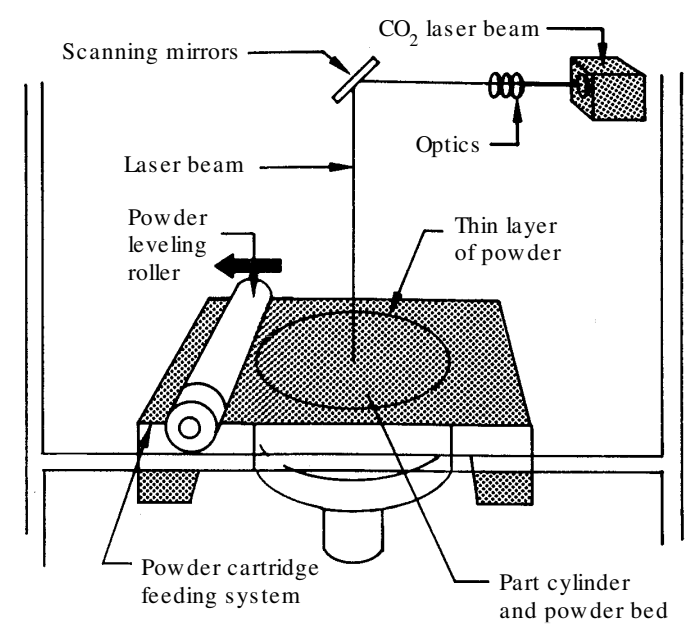

Thermal submodel

The heat transfer behavior in the powder bed can be described by the classical conduction equation:
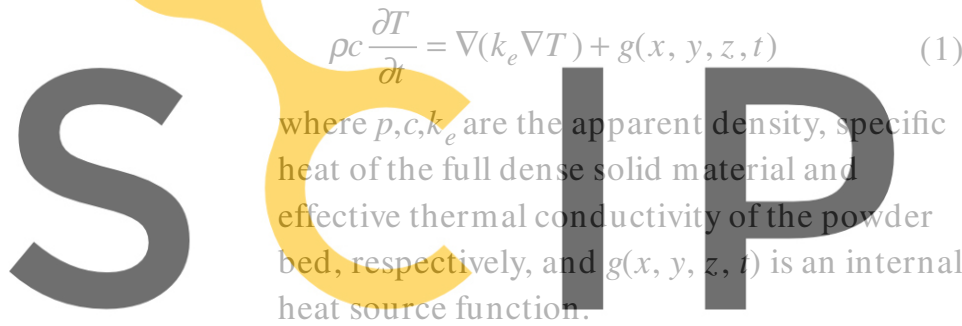

In contrast to the conduction problem of a

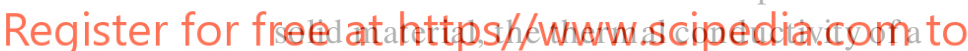
powder bed can vary from point to point

depending on the local temperature as well as the condition of contact between particles and the local porosity. Yagui and Kunni (1989) have formulated a model for the effective conductivity of a packed bed considering the conduction, convection and radiation effects within a powder bed:

$$
k_{e}=\frac{\mu k_{s}}{1+\Phi \frac{k_{s}}{k_{g}}}
$$

where $k_{s}$ is the conductivity of the solid material, $k_{g}$ is the conductivity of air, $\mu$ is the solid fraction $\mu=\frac{\rho}{\rho_{s}}$ (where $\rho_{s}$ is the density of the solid material) and $\Phi$ is an empirical coefficient normally taken as $\Phi=0.02 \times 10^{2(0.7-\mu)}$. Expression (2) allows to compute the real conductivity at each point of the problem in terms of the powder density.

The thermal problem to be solved with equation (1) is schematized in Figure 2.

There is a laser beam that moves in the $y$ direction with a constant velocity $v$. Owing to
Figure 2 Thermal model

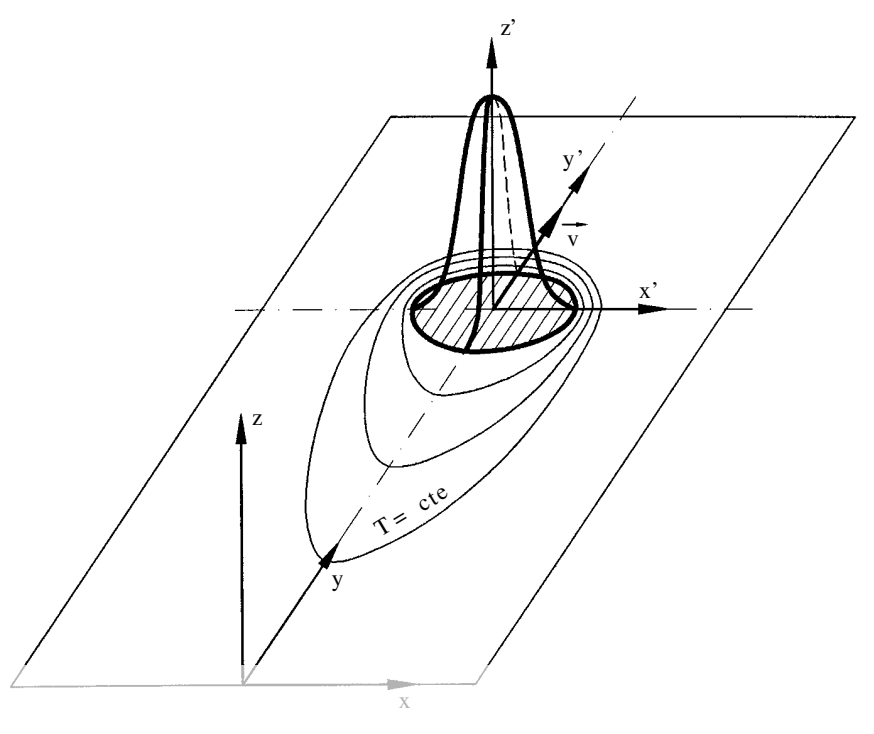

the movement of the laser beam this is a

transient problem. Nevertheless, it can be

transformed into a steady problem with a

change in the coordinate axes of the problem.

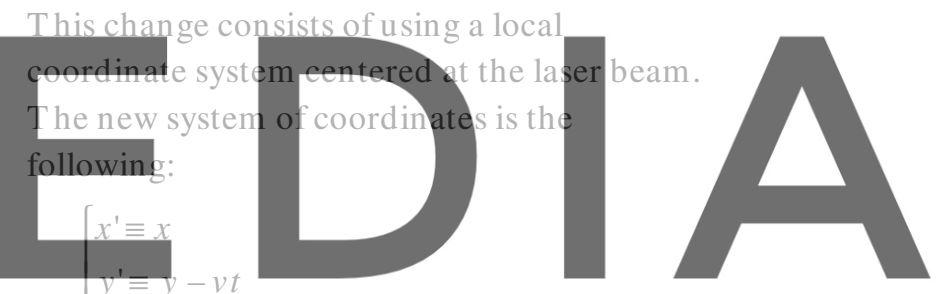

downlgad the version without the (3) $^{\prime} \equiv z$ atermark

Then equation (1) can be rewritten in terms

of the new coordinates system as:

$$
\rho c \frac{\partial T}{\partial y^{\prime}}=\nabla\left(k_{e} \nabla T\right)+g\left(x^{\prime}, y^{\prime}, z^{\prime}\right)
$$

From the mathematical point of view the main difference between equation (1) and equation (4) is that the last one does not contain any dependence with respect to the time variable $t$ but, on the other side, a new convective term appears at the left side of the equality. This type of term produces some additional difficulties.

In order to solve equation (4) the domain indicated in Figure 1 has been defined. Following the notation of Figure 1 and using $\boldsymbol{n}$ for the vector normal to a boundary and $\boldsymbol{q}$ for the thermal flux $\left(\boldsymbol{q}=k_{e} \nabla T\right)$ the boundary conditions that have been applied for the resolution of equation (1) are the following:

- Symmetry plane condition for $x^{\prime}=0$ $\left(\boldsymbol{n}^{t} \boldsymbol{q}=0\right)$. 
- Local temperature equal to the external temperature $\left(T=T_{e}\right)$ at $x^{\prime}=w, y^{\prime}=L_{1}$ and $z^{\prime}=-d$.

- Convection-radiation condition at $z^{\prime}=0$ : $\boldsymbol{n}^{t} \boldsymbol{q}=-\alpha\left(T-T_{e}\right)$ being $\alpha$ the convectiondiffusion coefficient.

- Thermal flux equal at the laser beam intensity $\left(\boldsymbol{n}^{t} \boldsymbol{q}=I\right)$ at the incidence zone.

- Owing to the fact that both the temperature and the thermal flux are unknown at $y^{\prime}=-L_{2}$ unless this part of the boundary be very far away from the laser beam no boundary condition has been applied there. From the mathematical point of view this is an unusual type of boundary condition. Additional information about this type of boundary condition can be found in (Papanastasiou et al., 1992).

\section{Sintering submodel}

To follow the evolution of the density (or void fraction) as a function of time and temperature, the Scherer (1977a; 1997b; 1977c;

1986) and the Mackenzie and Shuttleworth
(1949) models have been used. Both of these
models assume that the surface energy reduc-
tion of the sintering powder drives the process
through viscous mass flow dissipation. In the
lower density ran ge (i.e. $\rho \leq 0.94 \rho_{S}$ ) the
Scherer model assumes that the powder

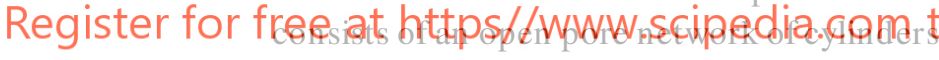
arranged cubically, with the cylinder diameters equal to the particle diameter and the cylinder lengths proportional to the pore diameter (see Figure 3). As sintering proceeds, the cylinder heights collapse, reducing the void fraction in the powder bed, until the

Figure 3 Scherer model for $\rho \leq 0.94 \rho_{s^{\prime}}$ open pore network of cylinders arranged cubically

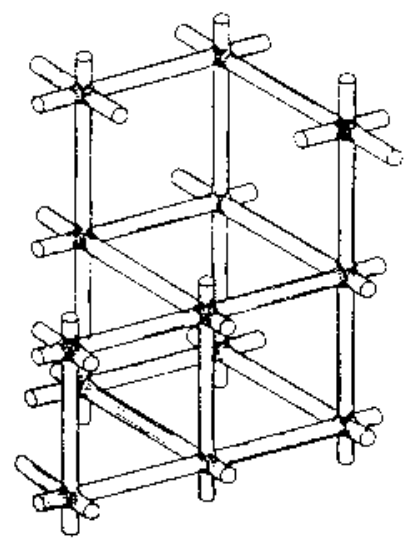

cylinder walls touch each other. At this point the Mackenzie-Shuttleworth model describes the powder bed by contiguous spheres with closed pores (see Figure 4).

The essential relationships for both models describe the free strain rate $\frac{\partial e}{\partial t}$ of the sintering material as follows:

$$
\frac{\partial e}{\partial t}=-\frac{M}{\eta} \frac{(3 \pi)^{1 / 3}}{6} \frac{2-3 d X}{\sqrt[3]{X(1-d X)^{2}}}
$$

for open pore, low density powder beds and partially sintered parts (i.e. $\rho \leq 0.94 \rho_{s}$ ) where $X$ is the cylinder aspect ratio (Scherer model), and

$$
\frac{\partial e}{\partial t}=-\frac{M}{\eta} \frac{1}{2} \frac{(4 \pi)^{1 / 3}}{3}\left(\frac{\rho_{s}}{\rho}-1\right)^{2 / 3}
$$

for closed pore beds (i.e. $\rho>0.94 \rho_{s}$ ) and solid parts (Mackenzie-Shuttleworth model). The quantity $M$ appearing in both equations is given by:

$$
M=\gamma n^{1 / 3}
$$

where $\gamma$ is the surface energy and $n$ is the

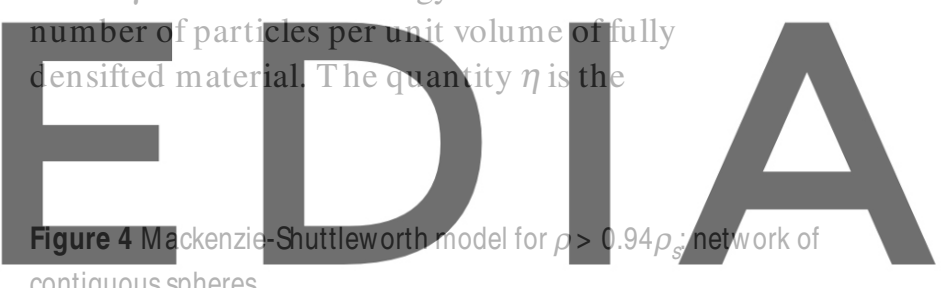

contiguous spheres

download the version without the watermark
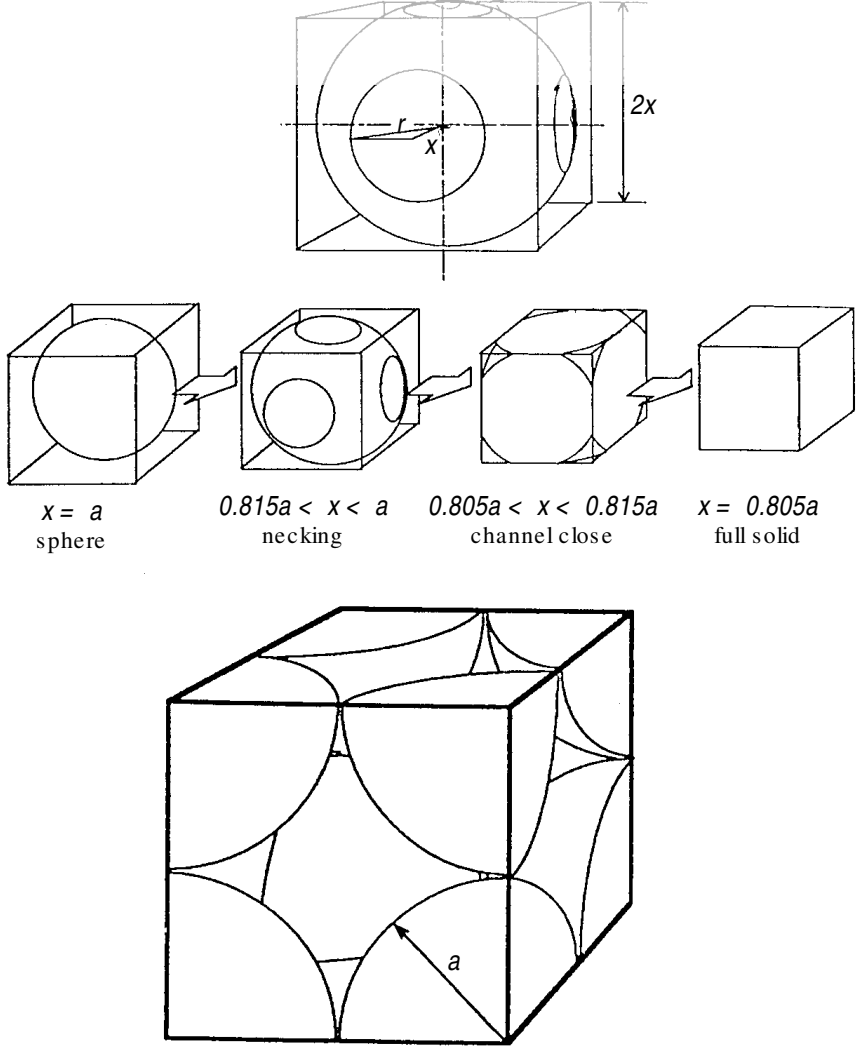
viscosity of the material, which is an Arrhenius-type function of temperature, given by:

$$
\eta=\eta_{0} \exp \left(\frac{\Delta E}{R T}\right)
$$

where $\eta_{0}$ is the viscosity coefficient and $\Delta E$ is the activation energy. The strain rate for low density sintering is also seen to be a function of $X$. This is the aspect ratio of the cylinders that make up the unit cell in that model. The constant $d$ in equation (5) is $8 \sqrt{2} / 3 \pi$. In the Mackenzie-Shuttleworth model, the strain rate is a function of the ratio of the densities of the fully sintered material $\rho_{s}$ and the actual density of the bed $\rho$.

As the particles sinter, the void fraction $\varepsilon$ drops. The Scherer model gives an expression for $\varepsilon$ as a function of the cylinder aspect ratio in the unit cell as:

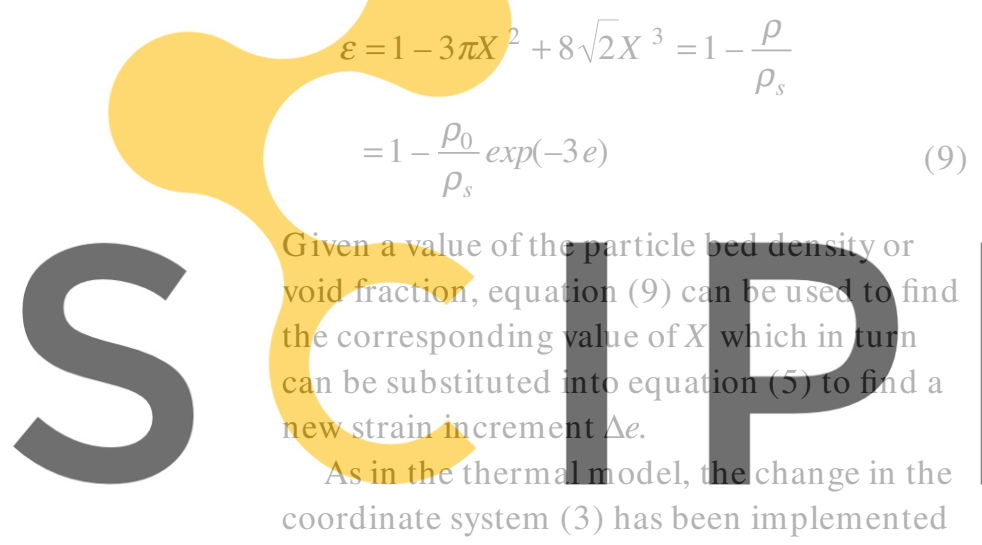

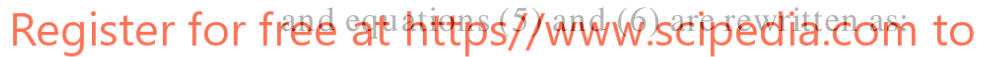

$$
\left\{\begin{array}{c}
\frac{\partial e}{\partial y^{\prime}}=\frac{M}{\eta} \frac{(3 \pi)^{1 / 3}}{6 v \quad \frac{2-3 d X}{\sqrt[3]{X}(1-d X)^{2}}} \\
\quad \text { if } \rho \leq 0.94 \rho_{s} \\
\frac{\partial e}{\partial y^{\prime}}=\frac{M}{\eta} \frac{1}{2 v} \frac{(4 \pi)^{1 / 3}}{3}\left(\frac{\rho_{s}}{\rho}-1\right)^{2 / 3} \\
\text { if } \rho>0.94 \rho_{s}
\end{array}\right.
$$

\section{Coupled model and solution strategy}

The analysis of the sintering process involves the coupled solution of the thermal equation (4) and the sintering equations (10). Both equations are linked by the expression (2) that relates the thermal conductivity at each point with the powder bed density. The strategy for the solution of this set of coupled equations is as follows:

(1) Assuming a given distribution of thermal conductivities equation (4) is solved by $\mathrm{u}$ sing a finite element discretization. This discretization leads to a non-symmetric system of equations. A stream upwind
Petrov Galerkin (SUPG) strategy (Brooks and Hughes, 1982) together with a shock capturing scheme (Codina, 1993) are used for the elimination of the spurious oscillations produced by the first order derivative term of equation (4).

(2) The nodal temperatures obtained in (1) are used for the obtainment of a new set of nodal density values. These new values are obtained by using equations (7), (8), (9) and (10).

(3) With the new density values equation (2) is used to get new values of the thermal conductivity. The new thermal conductivity values allow to go back to step (1). This iterative process is repeated until convergence.

\section{The necessary input data for this iterative solution process is: \\ - Laser beam intensity $I$, radius $w$ and beam velocity $v$ \\ Thermal properties: $k_{s}$ and $k_{d}$ \\ - Rheological properties $\mu_{0}, \Delta E, \eta_{0}$, surface tension $\gamma$.}

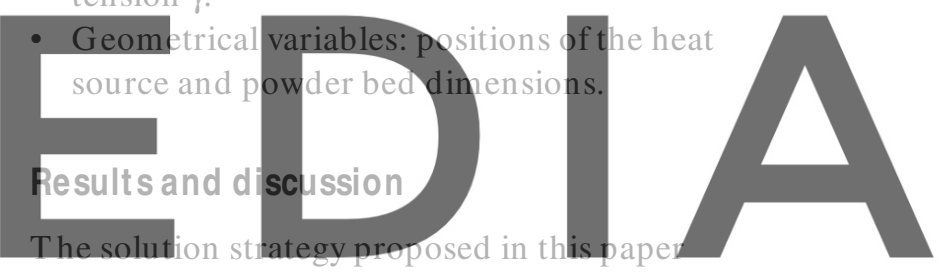

has been validated through the analysis of the

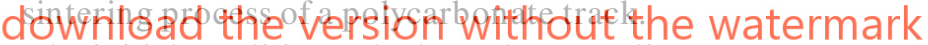 \\ The initial conditions, the boundary condi-}

tions, and the material properties are shown

in Table I. The finite element mesh shown in

Figure 5 with 115,616 nodes and 113,500

hexahedral eight noded elements has been used for the thermal analysis. Owing to the symmetry of the problem only one half of the domain has been discretized. The dimensions of this semi-domain are $0.2 \mathrm{~cm} \times 1.6 \mathrm{~cm} \times 0.2 \mathrm{~cm}$.

Table I Initial conditions, boundary conditions and material constants

Initial conditions:

Initial bed temperature $T_{\text {initial }}\left({ }^{\circ} \mathrm{K}\right)$

Initial density $\rho_{0}\left(\frac{\mathrm{kg}}{\mathrm{m}^{3}}\right)$

293 or 375

Initial solid fraction $\mu_{0}$

Material constants:

Full dense solid material $\rho_{s}\left(\frac{\mathrm{kg}}{\mathrm{m}^{3}}\right)$

Viscosity coefficient $\eta_{0}$ (Pa.s)

$5.41 \times 10^{-18}$

Activation energy $\Delta E\left({ }^{\circ} K\right)$

20638

Surface energy $\gamma\left(\Delta \mathrm{m}^{2}\right)$

$45 \times 10^{-5}$

Particle bed specific heat $C_{p}\left(V^{\circ} K k g\right)$

1,580

Conductivity of solid $K_{s}\left(W /{ }^{\circ} K . m\right)$

Conductivity of air $K_{g}\left(W /{ }^{\circ} K . m\right)$ 
Figure 5 Finite element mesh

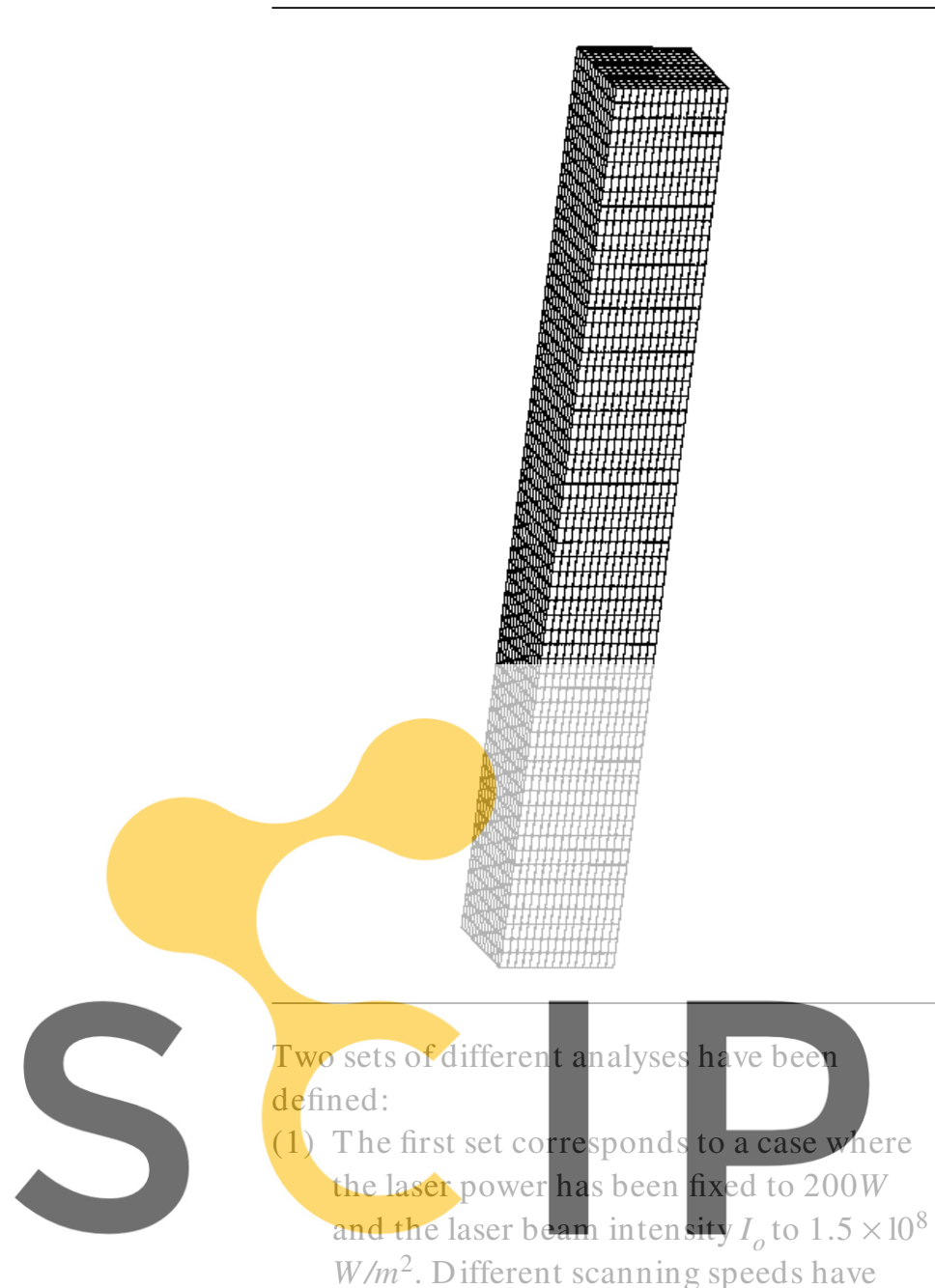

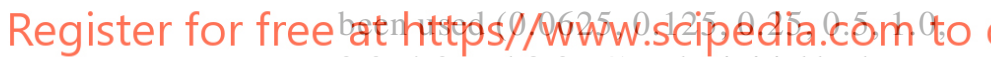
$2.0,4.0$ and $8.0 \mathrm{~m} / \mathrm{s}$ ). The initial bed

temperature is $293^{\circ} \mathrm{K}$. This corresponds

to a case where the initial polycarbonate powder has not been pre-heated and a high power laser beam has been used.

(2) The second set corresponds to a case where the laser power has been fixed to $10 \mathrm{~W}$ and the laser beam intensity $I_{o}$ to $5.0 \times 10^{7} \mathrm{~W} / \mathrm{m}^{2}$. Different scanning speeds have been used $(0.0625,0.125$, $0.25,0.5,1.0,2.0,4.0$ and $8.0 \mathrm{~m} / \mathrm{s})$. The initial bed temperature is $375^{\circ} \mathrm{K}$. This corresponds to a case where the initial polycarbonate powder has been preheated and, due to that, a lower power laser beam can produce a significant amount of sintering.

The objective of both sets of analyses has been to study how the sintered depth and the density distribution change with the variation of the scanning speed while the rest of sintering parameters are maintained fixed.

Figure 6 shows the obtained temperature distribution corresponding to a scanning
Figure 6 Temperature distribution for $\mathrm{v}=50 \mathrm{~cm} / \mathrm{s}$ and the $200 \mathrm{~W}$ laser power

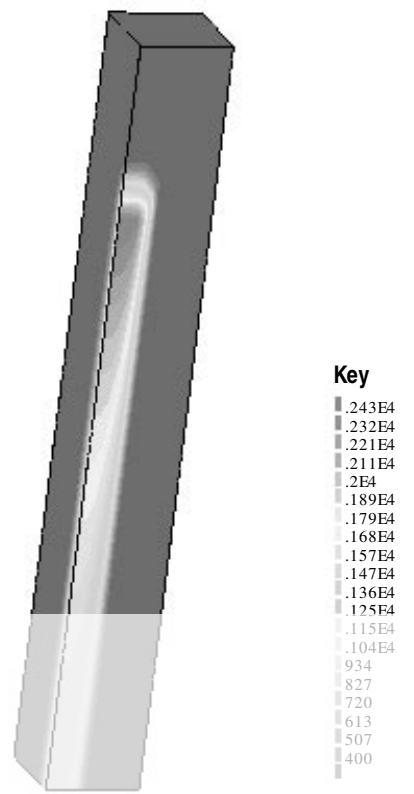

speed of $0.5 \mathrm{~m} / \mathrm{s}$ with a laser power of $200 \mathrm{~W}$.

Temperatures range from $293^{\circ} \mathrm{K}$, which is the

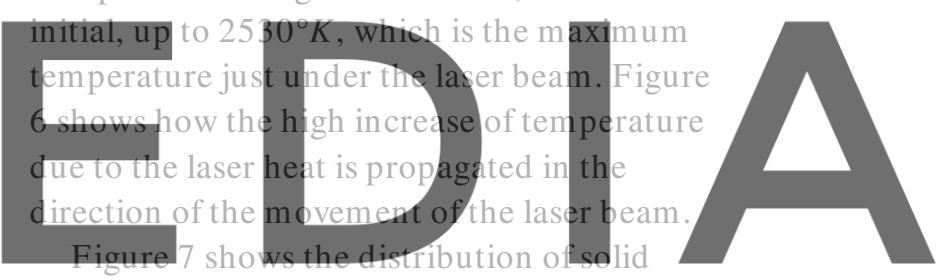

fraction $\mu$ in the powder bed corresponding to

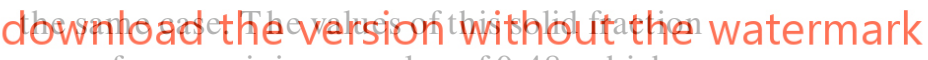
range from a minimum value of 0.48 , which corresponds to the initial density divided by

Figure 7 Solid fraction distribution for $v=50 \mathrm{~cm} / \mathrm{s}$ and the $200 \mathrm{~W}$ laser power

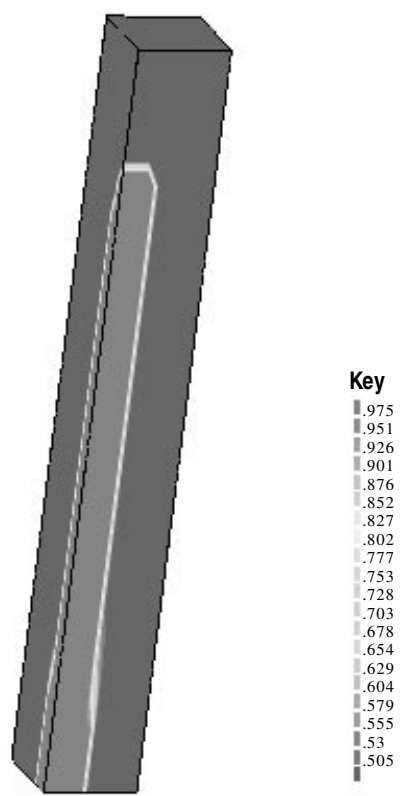


the density of the full solid material, up to 1.0, which corresponds to the completely sintered material. There is a very good definition of the zones where the material has not started to sinter $\left(\mu=\mu_{0}=0.48\right)$ and where it is completely sintered $(\mu=1.0)$. In addition, there is a very narrow band where the material is partially sintered which corresponds to solid fraction values ranging from 0.48 to 1.0 .

Figure 8 shows the sintered depth obtained for each scanning speed and for both sets of analyses. It shows that for velocities higher than a specific value the sintered depth is null and no sintered material is produced. On the other side, a decrease of the scanning speed produce an increase of the sintered depth. It also shows how the pre-heating of the initial powder bed allow to get sintered tracks with a much/lower laser power.

This results are in agreement with the experimentalones taken from Ming-Shen et al. (1991b).

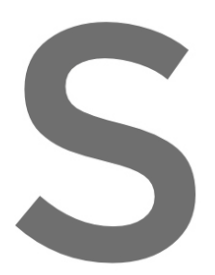

Conclusions
A three-dimension al
sintering is derived ba
structure: thermal an
In these models a new
been implemented to
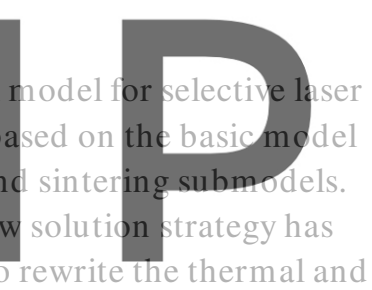

sintering equations in order to get a steady

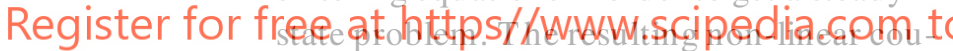

pled model converges in a small number of

iterations. The temperature and density

distributions obtained provide useful information about the sintering properties such as the sintering depth. Future goals of this work

Figure 8 Sintered depth for different scanning speeds for both sets of analysis

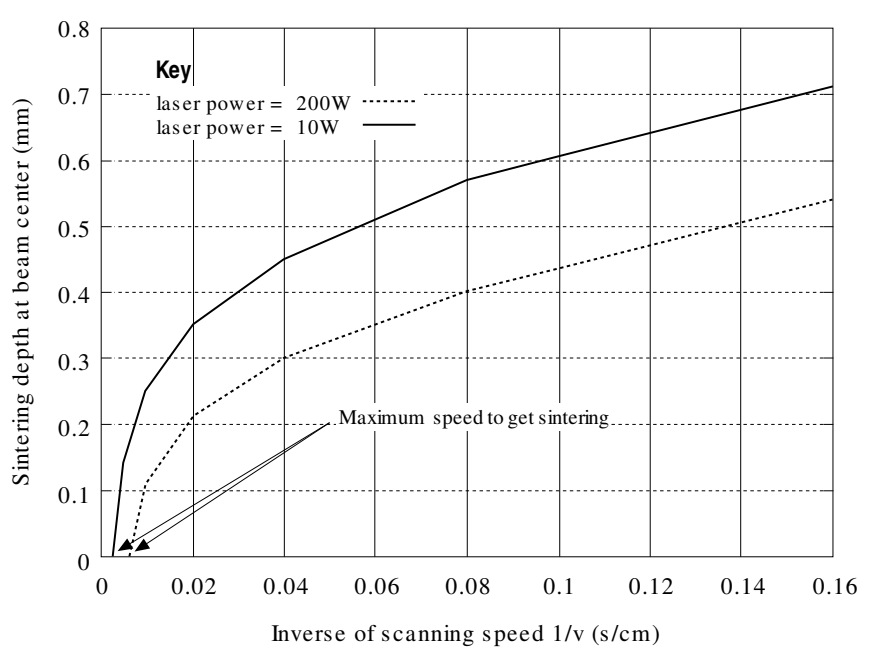

are to explore more experimental data on material properties to support each submodel and to obtain the residual stresses distribution.

\section{References}

Berzins, M., Childs, T.H.C., Dalgarno, K.W., Ryder, G.R. and Stein, G. (1996), "The selective laser sintering of polycarbonate", Annals of CIRP, Vol. 45 Part 1 , pp. 187-90.

Brooks, A.M. and Hughes, T.JR. (1982), " Streamline upwind/Petrov-Galerkin formulations for convection dominated flows with particular emphasis on the incompressible Navier-Stokes equation", Computer Methods in Applied Mechanics and Engineering, Vol. 32, pp. 199-259.

Codina, R. (1993), "A discontinuity-capturing crosswinddissipation for the finite element solution of the convection - diffusion equation", Computer Methods in Applied Mechanics and Engineering, Vol. 110, pp. 325-42.

Mackenzie, J and Shuttlew orth, R. (1949), "Phenomenological theory of sintering", Proc. Phys. Soc., Loudon, Vol. 62 [12-B], pp. 833-52.
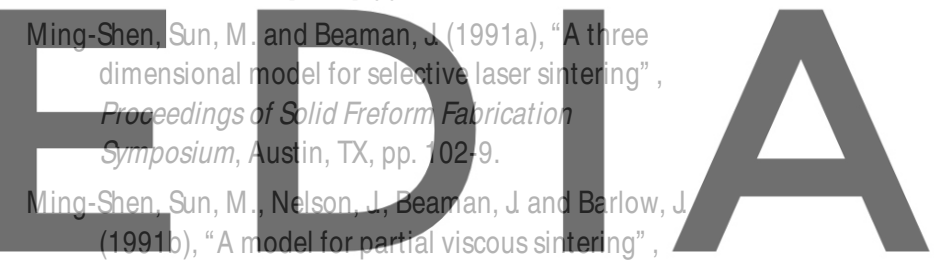
Proceedings of Solid Freform Fabrication

\section{downimpasigm the vertin, TX.} Papanastasiou, T.C., Malamataris, N. and Ellwood, K. (1992), "A new outflow boundary condition" international journal for Numerical Miethods in Fluids, Vol. 14, pp. 587-608.

Scherer, G. (1977a), "Sintering of low density glasses: I, theory”, J. Am. Cer. Soc., Vol. 60 No. 5-6, pp. 236-9.

Scherer, G. (1977b)m "Sintering of low density glasses: II, experimental study", J. Am. Cer. Soc., Vol. 60 No. 5-6, pp. 239-45.

Scherer, G. (1977c), "Sintering of low density glasses: III, effect of a distribution of pore size", J. Am. Cer. Soc., Vol. 60 No. 5-6, pp. 245-8.

Scherer, G. (1986), "Viscous sintering under a uniaxial load”, J. Am. Cer. Soc., Vol. 69 No. 9, pp. 206-07.

Weissman, E.M. and Hsu, M.B. (1991), "A finite element model of multilayered laser sintered parts", Proceedings of Solid Freform Fabrication Symposium, Austin, TX, pp. 86-94.

Williams, J.D. and Deckard C.R. (1998), "Advances in modeling the effects of selected parameters on the SLSprocess", Rapid Prototyping Journal, Vol. 4 No. 2, pp. 90-100.

Yagui, S. and Kunni, D. (1989), "Studies on effective thermal conductivities in packed beds" , J. AlChe., Vol. 3 No. 3, pp. 373-81. 